\section{Promising novel therapeutic approaches in the management of gastrointestinal stromal tumors}

\author{
Zoltan Szucs', Khin Thway', Cyril Fisher', Ramesh Bulusu², Anastasia Constantinidou', \\ Charlotte Benson', Winette TA van der Graaf ${ }^{1,3}$ \& Robin L Jones ${ }^{*, 1}$
}

Primary and secondary resistance to currently available licensed tyrosine kinase inhibitors poses a real clinical challenge in the management of advanced gastrointestinal stromal tumors. Within the frame of early phase clinical trials novel systemic treatments are currently being evaluated to target both the well explored and novel emerging downstream effectors of KIT and PDGFRA signaling. Alternative therapeutic approaches also include exploring novel inhibitors of the KIT/PDGFRA receptors, immune checkpoint and cyclin-dependent kinase inhibitors. The final clinical trial outcome data for these agents are highly anticipated. Integration of new diagnostic techniques into routine clinical practice can potentially guide tailored delivery of agents in the treatment of a highly polyclonal, heterogeneous disease such as heavily pretreated advanced gastrointestinal stromal tumor.

First draft submitted: 16 April 2016; Accepted for publication: 29 July 2016; Published online: 7 September 2016

The KIT tyrosine kinase receptor plays a vital role in the pathogenesis of gastrointestinal stromal tumors (GISTs) and therefore has become a universal therapeutic target. Imatinib is the first choice drug for the treatment of advanced/metastatic GISTs, four out of five patients clearly benefiting from treatment with a median overall survival (OS) of approximately 50 months [1]. While majority of GIST patients respond to imatinib treatment, approximately $10-15 \%$ of them show primary resistance with a further $40-50 \%$ developing secondary resistance to the agent with a median time to progression of about 24 months [2]. Sunitinib and regorafenib have been approved for the treatment of imatinib-resistant GIST, with far less impressive clinical efficacy and more disadvantageous toxicity profile as compared with imatinib $[3,4]$.

Ongoing preclinical and clinical research has provided powerful tools in the explanation, prediction and management of primary resistance. A strong link has been established between mutational status and sensitivity to tyrosine kinase inhibitors (TKIs), for instance PDGFRA exon 18 D842Vmutant GISTs are unlikely to respond to imatinib [5]. The acquisition of secondary mutations in KIT or PDGFRA represents the most frequent mechanism of imatinib resistance in GIST [6,7]. We elaborate to great length on the relevance of specific genetic changes leading to primary and secondary treatment resistance in our twin-review written on the topic of genetic subtypes of GIST [8].

The objective of this manuscript is to highlight the most relevant and recent novel therapeutic attempts trying to overcome complex, mostly polyclonal resistance to currently available TKIs. We focus on promising, previously less discussed emerging therapeutics including inhibitors of the KIT/

\section{KEYWORDS}

- GIST • KIT • novel therapy

- PFGFRA 
PDGFRA receptor, drugs targeting dysregulated downstream signaling pathways, immune checkpoint inhibitors and cyclin-dependent kinase inhibitors (Table 1). Clinical trial data are mostly immature for these agents therefore final results are highly anticipated.

\section{Novel KIT/PDGFRA receptor inhibitors}

Even with newer licensed multitarget kinase inhibitors such as regorafenib, resistance develops over time. However, the oncogenic KIT dependency of GIST persists even after failure of standard treatment options as ATP-mimetic TKIs do seem to provide some clinical benefit in this setting. A number of alternative ATP mimetics (nilotinib, masitinib, sorafenib, dovitinib, pazopanib) have been explored in treatment-resistant GIST with disappointingly mixed results; none of the clinical trials with these agents have led to regulatory body licensing [28]. Ponatinib is one of the more promising ATP-competitive KIT-inhibitors that was tested against a variety of KIT-mutant GISTs. Unlike currently available approved KIT inhibitors, ponatinib has also shown activity against the KIT exon 17 D816mutant kinases [29]. In a Phase II trial of $45 \mathrm{mg}$ daily dose ponatinib in heavily pretreated GIST patients with KIT exon 11 mutated tumors [9] the clinical benefit rate (CBR) (complete remission [CR], partial remission [PR] or SD) at 16 weeks was 37\% (10/27) [30]. Another Phase II trial of the German Arbeitsgemeinschaft Internistische Onkologie Group is currently evaluating ponatinib at $30 \mathrm{mg}$ daily dose in imatinib-resistant GIST [10].

New classes of non-ATP mimetic (switch pocket kinase inhibitors, such as DP-2976) have shown in vitro activity and could represent a promising strategy in the fight against TKI resistance [31].

As a means to suppress drug-resistant cell clones, sequential administration, as well as rotation of TKIs are being evaluated. Trying to ameliorate the TKI addiction of heavily pretreated GISTs a currently open Phase Ib study explores the safety and tolerability of sunitinib alternating with regorafenib in participants progressing on all standard approved therapies (imatinib, sunitinib and regorafenib) [32].

\begin{tabular}{|c|c|c|c|c|c|}
\hline Target & Class of agent (specific activity) & Drug(s) & Trial/Phase (combination) & Results & Ref. \\
\hline \multirow[t]{6}{*}{ KIT/PDGFRA } & Multitargeted TKI (KIT exon 17 & Ponatinib & NCT01874665 Phase II & $37 \%$ CBR at 16 weeks & [9] \\
\hline & D816-mutant kinases) & & AIO-STS-0115 Phase II & Awaited & [10] \\
\hline & Multitargeted TKI (PDGFRA D842V) & Dasatinib & Phase II & $\begin{array}{l}32 \% \text { PR; } 21 \% \text { PFS at } \\
6 \text { months }\end{array}$ & [11] \\
\hline & & & NCT01643278 Phase I (+ipilimumab) & Awaited & [12] \\
\hline & Multitargeted TKI & Crenolanib & NCT01243346 Phase I/II study & $31 \%$ CBR & [13] \\
\hline & KIT D816V/PDGFRA D842V inhibitor & BLU285 & NCT02508532 Phase I & Awaited & [14] \\
\hline \multirow[t]{2}{*}{ PI3K } & PI3K inhibitor & BYL719 & NCT01735968 Phase I & Awaited & {$[15]$} \\
\hline & $\begin{array}{l}\text { Selective PI3K catalytic } p 110 \alpha \text { subunit } \\
\text { inhibitor }\end{array}$ & Buparlisib & Phase I & Awaited & [16] \\
\hline BRAF V600E & BRAF inhibitor & Vemurafenib & NCT02304809 Phase II & Awaited & [17] \\
\hline \multirow[t]{2}{*}{ MEK } & MEK inhibitor & Binimetinib & NCT01991379 Phase Ib/II (+imatinib) & $33 \% \mathrm{PR}$ & [18] \\
\hline & MEK1/MEK2 TKI & Trametinib & NCT02342600 Phase II (+pazopanib) & Awaited & [19] \\
\hline \multirow[t]{2}{*}{ MET } & $\begin{array}{l}\text { Dual MET and KIT small-molecule } \\
\text { inhibitor }\end{array}$ & Cabozantinib & Phase I & $\begin{array}{l}\text { Long-lasting SD as best } \\
\text { response }\end{array}$ & [20] \\
\hline & & & NCT02216578 Phase II & Awaited & [21] \\
\hline FGFR & Pan-FGFR inhibitor & BGJ398 & NCT02257541 Phase Ib/II (+imatinib) & Awaited & [22] \\
\hline IGF1R & IGF1R inhibitor & Linsitinib & NCT01560260 Phase II & $\begin{array}{l}45 \% \text { CBR; } 52 \% \text { PFS, } 80 \% \text { OS } \\
\text { at } 9 \text { months }\end{array}$ & [23] \\
\hline \multirow[t]{2}{*}{ HSP90 } & Nonansamycin HSP90 inhibitor & Onalespib & NCT01560260 Phase I & $36 \%$ CBR & [24] \\
\hline & & & NCT01294202 Phase II ( \pm imatinib) & Awaited & [25] \\
\hline \multirow[t]{2}{*}{ CTLA4 } & Anti-CTLA4 antibody & Ipilimumab & NCT01738139 Phase I (+imatinib) & Single PR & [26] \\
\hline & & & NCT01643278 Phase I (+dasatinib) & $\begin{array}{l}\text { Single durable SD for } \\
59+\text { weeks }\end{array}$ & [12] \\
\hline CDK & CDK4/6 inhibitor & Palbociclib & NCT01907607 Phase II & Awaited & [27] \\
\hline
\end{tabular}


The Australasian Gastro-Intestinal Trials Group in collaboration with the European Organisation for Research and Treatment of Cancer Scandinavian Sarcoma Group are currently evaluating whether alternating imatinib and regorafenib in the first-line treatment of advanced GIST would delay the onset of TKI resistance with the primary outcome measure of PFS at 24 months [33].

A Phase II Study with the novel human antiPDGFR $\alpha$ monoclonal antibody olaratumab in previously treated patients with unresectable and/or metastatic GIST [34] has been terminated early due to lack of efficacy.

\section{- PDGFRA D842V-mutant inhibition}

GISTs harboring the PDGFRA D842V mutation represent the overwhelming majority of GISTs with primary imatinib and sunitinib resistance, while exhibiting some moderate response to regorafenib. A number of novel agents are being tested with an enhanced potential of targeting this very specific receptor mutation, including dasatinib, crenolanib and BLU-285 (Table 1).

Dasatinib is an oral multitarget TKI with an enhanced binding affinity for KIT and PDGFR. In preclinical studies imatinib-resistant PDGFRA D $842 \mathrm{~V}$ or imatinib-sensitive PDGFRA (DeltaDIM842-844)-mutant GIST cells and cell lines were treated with dasatinib, sorafenib, nilotinib and IPI-504 at different concentrations. The effect of these agents on proliferation, survival and signaling was examined. Of these agents only dasatinib showed potent inhibition of the PDGFRA D842V isoform with an IC(50) value of $62 \mathrm{nmol} / 1$ [35]. In a Phase II trial assessing the antitumor activity of dasatinib in patients with advanced GIST who were refractory to imatinib and sunitinib the PR rate was $32 \%$ $(15 / 47)$ by Choi criteria and $21 \%$ patients $(10 / 47)$ were progression free $>6$ months [11]. Dasatinib is currently assessed in combination with ipilimumab for patients with advanced GISTs and other sarcomas within a Phase I trial [12].

Crenolanib is a unique type I small-molecule inhibitor of FLT3 and the PDGFR receptors (including the $\mathrm{D} 842 \mathrm{~V}$-mutated kinase). In preclinical studies crenolanib proved to be a potent inhibitor of imatinib-resistant PDGFRA kinases (D842I, D842V, D842Y, DI842-843IM and deletion I843). In an isogenic model system crenolanib exhibited a 135-fold increased activity against the D842V-mutant GIST as compared with imatinib [36].
In a most recent Phase I/II study [13] crenolanib proved to be the first and only TKI to show activity in PDGFRA D842V-mutant advanced GIST. In this early phase trial $2 / 16$ patients achieved a PR and 3/16 achieved SD, corresponding to a CBR of $31 \%$ (5/16 patients). More encouragingly seven patients remained on crenolanib for over 6 months and one patient each for 1 year and 2 years, respectively. Crenolanib was well-tolerated when given to patients on a chronic basis. Only four out of 20 PDGFRAmutant GISTs were ${ }^{18} \mathrm{~F}$-fluorodeoxyglucose avid on baseline PET imaging [13]. A randomized placebo-controlled study of crenolanib in advanced D842V GIST has accordingly been initiated.

BLU285 is a mutation-specific inhibitor of KIT D816V and PDGFRA D842V mutated kinases conferring resistance to most currently available TKIs [37]. In a TKI-resistant KIT exon 11/17-mutant GIST patient derived xenograft model the BLU-285 compound showed dosedependent marked inhibition of tumor growth, proliferation, KIT signaling and induction of apoptosis. At the $30 \mathrm{mg} / \mathrm{kg}$ dose BLU-285 treatment resulted in striking tumor regression with a $73 \%$ reduction of baseline measurements. At the same $30 \mathrm{mg} / \mathrm{kg}$ dose BLU-285 treatment led to a 3.4-fold increase in apoptosis as compared with control. At a lower $10 \mathrm{mg} / \mathrm{kg}$ dose BLU285 stabilized the tumor volume led to a 27 -fold decrease in the proliferative index. Treatment with both doses decreased significantly the activity of pMAPK and KIT signaling [38].

A Phase I trial of this orally administered agent is currently open to enrollment for adult patients with advanced GIST and other solid tumors [14]. The dose expansion component of the trial includes a cohort of patients with D $842 \mathrm{~V}$ mutated tumors. If successful as a single agent, with its very narrow inhibition profile [37] BLU285 could become in the future a candidate for combination trials.

Inhibition of downstream signaling pathways KIT/PDGFRA-mutant GISTs feature oncogenic signaling via both the PI3K/mTOR and RAS/MAPK pathways. Li and colleagues tried to further elucidate the biological and clinical relevance of these pathways in GISTs that lose KIT/PDGFRA dependence [39]. Their 17 patient study included patients with high-risk or metastatic GIST that were either KIT/PDGFRA/SDH wild-type or KIT-mutant with progression on TKI but no secondary KIT mutation. They 
also developed GIST cell lines to assess the biologic role and clinical implications of PI3K and/or RAS pathway oncogenic activation. Eight GISTs had mutations activating the PI3K and/or RAS pathways, seven with both PI3K and RAS pathway derangements. The KIT-mutant GIST882 sublines with PTEN and NF1 inactivation, or with NF1 inactivation alone, were imatinib-resistant, whereas parental GIST882 and a subline with PTEN inactivation alone remained imatinib-sensitive. A novel GIST line, NS72, with NF1 inactivation and both PIK3CA and PTEN mutations was imatinib-resistant. These findings prove that co-activation of the RAS and PI3K pathways in GIST fosters KITindependence and contributes to TKI resistance. While RAS pathway activation in GIST models leads to imatinib resistance, PI3K pathway activation alone does not. Continued efforts should focus on developing cotargeting strategies for the RAS and PI3K pathways in GIST.

Van Looy and colleagues tested the in vivo efficacy of three PI3K inhibitors (PI3Ki) in patient-derived GIST xenograft models carrying diverse KIT genotypes and PTEN genomic status [40]. The studied oral PI3Kis were buparlisib (BKM120) a pan-PI3Ki, BEZ235 - a dual pan PI3K/mTOR inhibitor and BYL719 - a selective inhibitor of the PI3K catalytic p110 $\alpha$ subunit. PI3Ki monotherapy led to significant tumor volume reduction or stabilization, mitotic activity and PI3K signaling inhibition. Combining imatinib with PI3Kis showed a marked synergistic antitumor activity. Response to the imatinib-PI3Ki combination was found dependent on the KIT genotype and specific model-related molecular characteristics. In the light of their results the authors suggested KIT genotype driven patient selection for clinical trials exploring such combinations.

A Phase I study was performed to determine the maximum tolerated dose and/or recommended Phase II dose of a combination of imatinib and the selective inhibitor of the PI3K catalytic p110 $\alpha$ subunit BYL719 in the third-line treatment of GIST patients [15]. This study is ongoing, but closed to further recruitment. Results of a closed Phase Ib dose-finding study [16] with the pan-PI3Ki BKM120 (buparlisib) in combination with imatinib in patients with GIST who have failed prior therapy with imatinib and sunitinib are eagerly awaited.

Alternate signaling pathway mutations, such as $B R A F$ exon 15 activating mutations can be one of the potential reasons for primary imatinib resistance [41]. The BRAF inhibitor dabrafenib showed some promising efficacy in BRAF V600E-mutant GIST, however, in the context of a single patient case report [42]. More efforts shall be focused on exploring the activity of BRAF inhibitors in this select population within the frame of prospective clinical trials. In order for easy access to vemurafenib for patients with $B R A F$-mutant tumors, the French National Cancer Institute (INCa) launched the AcSé V program [17], funding both access to molecular diagnosis in the 28 INCa molecular genetic centers and an exploratory Phase II trial testing the drug. Interestingly patients with $B R A F$ non-V600 mutations (on exon 11 or 15) or other $B R A F$ alterations identified through a pan-genomic tumor profile are also eligible and included into a miscellaneous cohort. The project aims to perform around 3000 molecular tests and to recruit up to 500 patients from 150 centers over 3 years.

Further novel systemic approaches are currently being evaluated in targeting the well explored and novel emerging downstream effectors of KIT and PDGFRA signaling, which we discuss below.

\section{ETV1/MEK inhibition}

The ETS (E 26) family transcription factor ETV1 shows high protein and mRNA level expression in GIST, and is essential for tumor growth and survival in both imatinib-sensitive (GIST882) and imatinib-resistant (GIST48) cell lines [43]. Activating KIT mutations cooperate with ETV1, the cellular levels of which are controlled by the KIT MAPK3/1 (ERK1/2) cascade to bring about GIST oncogenesis [43,44]. Under basal conditions, oncogenic and/or wildtype KIT and wild-type PDGFRA cooperatively activate ERK, thereby preventing ETV1 degradation. High levels of ETV1 stimulate cell proliferation and tumorigenesis by hyperactivating ICC/GIST-specific transcriptional output, including KIT expression [45]. In KIT-mutant GIST, inhibition of PDGFRA disrupts the KIT-ERK-ETV1-KIT signaling loop by inhibiting ERK activation and facilitating ETV1 degradation. Reduced ETV1 levels limit cell proliferation via reduced transcriptional activation of target genes including $K I T$ [45].

Considering the role of ETV1 as a master regulator of the ICC lineage, required for GIST initiation and proliferation, it has been considered 
as a new key therapeutic target [43-45]. The dual lineage targeting of KIT by imatinib and ETV1 by the MEK inhibitor MEK162 induced more apoptosis than single-agent imatinib or MEK162 in human GIST cells. The combination therapy resulted in complete tumor regression, whereas single-agent imatinib or MEK162 treatment led to disease stabilization in human GIST xenograft studies. Moreover, combination therapy also induced more tumor fibrosis than single-agent imatinib or MEK162 treatment in genetically engineered mouse models of GIST [44].

The combination of imatinib and the MEK inhibitor/binimetinib is currently investigated in the first-line treatment of advanced GIST patients. In a Phase Ib/II trial [18] for a heavily pretreated patient population, nine out of the 15 evaluable patients treated with the combination had stable disease at 8 weeks and five had a partial response according to the Choi criteria.

Trametinib is a MEK1/MEK2 kinase inhibitor indicated for the treatment of patients with unresectable or metastatic melanoma with $B R A F \mathrm{~V} 600 \mathrm{E}$ or V600K mutations. A Phase II pilot trial [19] is to assess the efficacy of trametinib in combination with pazopanib in imatinib/sunitinib-refractory advanced GIST patients.

\section{MET signaling inhibition}

The acquired expression of activated forms of the $M E T$ oncogene was observed in human GIST specimens that acquired imatinib resistance. Similar MET activation also developed after imatinib therapy in a mouse model of GIST (KitV558del/+ mice) and in imatinib-sensitive human GIST cell lines after imatinib treatment in vitro. The dual MET and KIT small-molecule inhibitor cabozantinib proved to be markedly more effective than imatinib in multiple preclinical models of both imatinib-sensitive and imatinib-resistant GIST [46].

In a Phase I trial cabozantinib administered $60 \mathrm{mg}$ daily appeared to be well tolerated and antitumor activity was observed in heavily pretreated GIST patients with long-lasting SD as best response [20]. A multicenter, multinational, open label, single arm Phase II study of single-agent cabozantinib is to be opened by the European Organisation for Research and Treatment of Cancer. The study [21] will be assessing the safety and activity of cabozantinib in patients with metastatic GIST who have previously progressed on imatinib and sunitinib and have not been exposed yet to other KIT - or PDGFR-directed TKIs.

\section{- FGFR inhibition}

Gene expression data have revealed that FGF2 and FGFR1 are overexpressed in all primary GIST samples examined, suggesting that FGFR signaling may limit imatinib's efficacy [47]. Combination of imatinib with BGJ398, a potent and selective small-molecule inhibitor of FGFR 1-3 increased growth inhibition in imatinib-sensitive GIST cell lines (an effect observed in the presence or absence of added FGF2) and enhanced efficacy in patient-derived GIST xenografts. In the absence of added FGF ligands, prolonged exposure of KIT-mutant GIST cells to imatinib was associated with ERK signaling reactivation. The ERK reactivation was further accompanied by FGFR activation, while the ERK rebound was repressed by the FGFR inhibitor BGJ398. It seems imatinib treatment induces feedback activation of FGFR signaling that can attenuate the antitumor effects of imatinib [47]. These preclinical results provided a rationale for combining imatinib and FGFR inhibitors, such as BGJ398, in the first-line therapy of GIST. A current trial [22] evaluates the pan-FGFR inhibitor BGJ398 in combination with imatinib in untreated advanced GIST, with no published results yet available.

\section{- IGF1R inhibitors}

In succinate dehydrogenase deficient $K I T /$ PDGFRA wild-type GISTs upregulation of HIF1- $\alpha$ may lead to increased growth signaling through the IGF1R and the VEGFR $[48,49]$. $I G F 1 R$ amplification may itself represent another mechanism of de novo or acquired imatinib resistance. A Phase II Study [23] evaluated the efficacy of IGF1R inhibitor linsitinib (OSI906) in pediatric and adult KIT/PDGFRA wild-type. All 20 eligible patients in the stage I of the study were adults and had advanced KIT/PDGFRA wild-type GIST. Treatment with linsitinib was well tolerated. While no objective responses were seen, qualitative partial and stable ${ }^{18} \mathrm{~F}$-fluorodeoxyglucose metabolic responses were seen in 6/17 (35\%). CBR (CR, $\mathrm{PR}$ and $\mathrm{SD} \geq 9$ months) at 9 months was $45 \%$. Kaplan-Meier estimates were $52 \%$ for PFS and $80 \%$ for OS at 9 months.

\section{- HSP90 inhibitors}

TKI-resistant KIT oncoproteins seem to require HSP90 chaperoning and thus are potently 
inactivated by HSP90 inhibitors. However, there are constrains to their clinical application by significant toxicity resulting from concomitant inactivation of various other HSP90 client proteins [28].

Onalespib (AT13387), a small molecule inhibitor of HSP90 showed promising clinical activity in GIST in a Phase I clinical trial, one patient had PR lasting for 10 months, and three had SD for up to 8 months [24]. The promising results of the Phase I trial prompted initiation of a Phase II trial in GIST [25], but the results are still pending. A recent publication [50] highlighted the role of CDC37 as a crucial HSP90cofactor in both imatinib-sensitive and imatinibresistant GIST. Targeting CDC37 is expected to be KIT/PDGFRA selective and represents a promising future strategy for inactivating KIT/PDGFRA oncoproteins in TKI-resistant GIST patients.

\section{Immune checkpoint inhibitors}

The immune system represents a swiftly emerging therapeutic target in all solid tumors. The PD-1/PD-L1 pathway is a key player in inhibiting the anticancer immune response. More recently anti-PD1 and anti-PDL1 drugs showed increasingly promising results in patients with solid tumors such as lung cancer and melanoma [51,52].

There are very little published data available on the expression of checkpoint proteins such as PD1/PDL1 in GIST. DNA microarray analysis for PDL1 expression in clinical samples of 139 operated imatinib-untreated localized GISTs [53] showed a heterogeneous PDL1 expression. PDL1 expression values varied over three decades on the logarithmic scale, providing the opportunity to search for histopathological-clinical feature correlations. In multivariate analysis, the PD-L1-low group was associated with a higher metastasizing risk, independent from clinicopathologic risk stratification and KIT mutational status.

The SARC trial evaluated pembrolizumab, an antibody that targets the PD-1 receptor, in advanced sarcomas [54]. Unfortunately, while the trial entry criteria had not specifically excluded GIST patients none were enrolled to the study.

In a murine model of spontaneous GIST it was found that the immune system contributes substantially to the antitumor effects of imatinib [55]. Imatinib therapy activated the $\mathrm{CD}^{+} \mathrm{T}$ cells and induced Treg apoptosis within the murine tumor by reducing tumor cell expression of the immunosuppressive enzyme indoleamine 2,3-dioxygenase. Moreover, concurrent immunotherapy with CTLA-4 blockage enhanced imatinib activity in murine GIST. In freshly obtained human GIST specimens, the T-cell profile showed a correlation with imatinib sensitivity and indoleamine 2,3-dioxygenase expression. $\mathrm{T}$ cells seem to play a crucial part in the antitumor effects of imatinib in GIST.

Concomitant immunotherapy given alongside targeted agents could synergistically enhance antitumor T-cell activation, thus improving outcomes in the treatment of solid tumors. In a recent Phase I trial [26] combination therapy of imatinib and ipilimumab immunotherapy was explored in metastatic or unresectable solid tumors. Among the 26 patients three objective responses were seen, in one GIST (PR) and two melanoma patients $(\mathrm{CR}+\mathrm{PR})$, respectively. Notably, both melanoma responders had $K I T$ mutations, while the GIST responder was of KIT/PDGFRA wild-type. Responders in this trial suggested that this combination at maximum tolerated dose has antitumor activity in KIT/PDGFRA wild-type GIST and KIT-mutant melanoma and merits further investigation.

Early results of a still recruiting trial (NCT01643278) investigating the combination of the dasatinib and ipilimumab (anti-CTLA-4 antibody) were promising with one out of the eight GIST patients treated showing a durable SD for 59+ weeks [12].

\section{- Cyclin-dependent kinase inhibitors}

$C D K N 2 A$ (coding for the $16^{\mathrm{INK} 4 \mathrm{a}}$ tumor suppressor protein) loss is a common genetic aberration in metastatic GIST [56]. The prognostic power of a 67 gene expression signature related to genome complexity (Complexity INdex in SARComas - CINSARC) was evaluated in GISTs. p16 $(C D K N 2 A)$ and retinoblastoma $(R B 1)$ gene deletions were likely causal events leading to increased CINSARC gene expression, chromosome rearrangement and ultimately development of metastasis [57]. Low p16 ${ }^{\mathrm{INK} 4 \mathrm{a}}$ expression was associated with response to the cyclin-dependent kinase inhibitor PD-0332991 in several in vitro tumor models [58]. Considering the preclinical data a Phase II trial of PD-0332991 (palbociclib) was initiated in advanced GIST patients refractory to imatinib and sunitinib with comparative genomic hybridization confirmed alteration 
of p16 $6^{\mathrm{INK} 4 \mathrm{a}}$. This study is currently recruiting participants [27].

\section{Conclusion}

The efficacy of standard treatment options to control advanced GIST is inevitably limited by resistance. Ongoing preclinical and clinical research is focusing on evaluating novel therapeutic approaches to overcome primary and secondary resistance to imatinib and the other two currently available licensed medications, sunitinib and regorafenib. Targeting deregulated downstream pathways shall provide further treatment options in the management of imatinib/sunitinib/regorafenibinsensitive/resistant GISTs.

\section{Future perspective}

Repeat biopsy genotype analysis in TKI-resistant GIST is limited by intra- and interlesional mutational heterogeneity of secondary mutations during the course of treatment. To overcome these limitations blood-derived circulating tumor DNA can be used in the future as biomarkers for prediction of treatment response. Identifying resistance mutations in plasma DNA would allow early switch to alternative TKIs or dose escalation of imatinib for optimal disease control [59]. Results of the Phase III GRID trial [60] were encouraging as $84 \%$ concordance was found between plasma and tissue for detection of primary KIT mutations. However, the assay was less sensitive for the detection of primary KIT exon 11 mutations in plasma DNA. These discrepancies in part might be attributed to the extensive heterogeneity of primary KIT exon 11 mutations and the difficulty to develop specific assays for each possible mutation. Bearing in mind its' potential limitations, further optimization of 'liquid biopsy' as a routine clinical diagnostic technique is certainly a promising path to follow.

\section{Financial \& competing interests disclosure}

The authors have no relevant affiliations or financial involvement with any organization or entity with a financial interest in or financial conflict with the subject matter or materials discussed in the manuscript. This includes employment, consultancies, honoraria, stock ownership or options, expert testimony, grants or patents received or pending, or royalties.

No writing assistance was utilized in the production of this manuscript.

\section{Open access}

This work is licensed under the Creative Commons Attribution-NonCommercial 4.0 Unported License. To view a copy of this license, visit http://creativecommons.org/ licenses/by-nc-nd/4.0/

\section{EXECUTIVE SUMMARY}

\section{Novel treatment options}

- Ponatinib is a promising ATP-competitive KIT inhibitor under further clinical evaluation.

- The multitargeted tyrosine kinase inhibitor (TKI) dasatinib and crenolanib have showed promising results in the treatment of PDGFRA D842V-mutant gastrointestinal stromal tumor (GIST).

- Phase I trial results with the promising KIT D816V/PDGFRAD842V-mutant-specific inhibitor BLU-285 are eagerly awaited.

- The BRAF inhibitor dabrafenib showed therapeutic efficacy in BRAF-mutant GIST, awaiting further clinical evaluation.

- The ETS family transcription factor ETV1 is universally highly expressed in GIST. Dual lineage targeting of KIT by imatinib and ETV1 by the MEK inhibitor binimetinib is currently evaluated in a Phase lb/ll clinical trial.

- Acquired expression of activated forms of the MET oncogene was observed in human GIST specimens that acquired imatinib resistance. Cabozantinib, a dual MET and KIT small-molecule inhibitor has already shown some efficacy in a Phase I clinical trial, awaiting further evaluation.

- FGF2 and FGFR1 are highly expressed in all primary GISTs. The pan-FGFR inhibitor BGJ398 in combination with imatinib is currently evaluated in untreated advanced GIST.

- Targeting upregulated IGF1R expression with linsitinib has already shown promising clinical activity.

- The immune system represents an emerging therapeutic target in all solid tumors, including GIST. The anti-CTLA-4 antibody ipilimumab is currently assessed in combination with TKIs for the treatment of advanced GISTs.

- The efficacy of the cyclin-dependent kinase inhibitor palbociclib is assessed in advanced GIST patients refractory to imatinib and sunitinib. 


\section{References}

Papers of special note have been highlighted as: - of interest

1 Gastrointestinal Stromal Tumor Meta-Analysis Group (MetaGIST). Comparison of two doses of imatinib for the treatment of unresectable or metastatic gastrointestinal stromal tumors: a meta-analysis of 1,640 patients. J. Clin. Oncol. 28(7), 1247-1253 (2010).

2 Van Glabbeke M, Verweij J, Casali PG et al. Initial and late resistance to imatinib in advanced gastrointestinal stromal tumors are predicted by different prognostic factors: a European Organisation for Research and Treatment of Cancer-Italian Sarcoma Group-Australasian Gastrointestinal Trials Group study. J. Clin. Oncol. 23(24), 5795-5804 (2005).

3 Demetri GD, van Oosterom AT, Garrett CR et al. Efficacy and safety of sunitinib in patients with advanced gastrointestinal stromal tumour after failure of imatinib: a randomised controlled trial. Lancet 368(9544), 1329-1338 (2006).

4 Demetri GD, Reichardt P, Kang Y et al. Efficacy and safety of regorafenib for advanced gastrointestinal stromal tumours after failure of imatinib and sunitinib (GRID): an international, multicentre, randomised, placebo-controlled, Phase 3 trial. Lancet 381, 295-302 (2013).

5 Heinrich MC, Owzar K, Corless CL et al. Correlation of kinase genotype and clinical outcome in the North American Intergroup Phase III Trial of imatinib mesylate for treatment of advanced gastrointestinal stromal tumor: CALGB 150105 Study by Cancer and Leukemia Group B and Southwest Oncology Group. J. Clin. Oncol. 26(33), 5360-5367 (2008).

6 Gramza AW, Corless CL, Heinrich MC. Resistance to tyrosine kinase inhibitors in gastrointestinal stromal tumors. Clin. Cancer Res. 15(24), 7510-7518 (2009).

7 Liegl B, Kepten I, Le C, Zhu M et al. Heterogeneity of kinase inhibitor resistance mechanisms in GIST. J. Pathol. 216(1), 64-74 (2008).

8 Zoltan Szucs, Khin Thway, Cyril Fisher et al. Molecular subtypes of gastrointestinal stromal tumors and their prognostic and therapeutic implications. Future Oncol. doi:10.2217/fon-2016-0192 (2016) (Epub ahead of print).

9 ClinicalTrials.gov Identifier: NCT01874665. 45 mg Daily Dose Phase 2 Trial of Ponatinib in Patients With Metastatic and/or Unresectable Gastrointestinal Stromal Tumor Following Failure of Prior Tyrosine Kinase
Inhibitor Therapy.

https://clinicaltrials.gov

10 AIO-STS-0115: Phase 2 Trial of Ponatinib in Patients with Metastatic and/or Unresectable Gastrointestinal Stromal Tumor (GIST) following Failure of Prior Therapy with Imatinib (POETIG trial - Ponatinib after rEsisTance to Imatinib in GIST). www.aio-portal.de

11 Trent K, Wathen M, von Mehren B et al. Sarcoma Alliance for Research through Collaboration: a Phase II study of dasatinib for patients with imatinib-resistant gastrointestinal stromal tumor (GIST). J. Clin. Oncol. 29(Suppl.) Abstract 10006 (2011).

- Shows promising early results with dasatinib.

12 Shoushtari AN, D’Angelo SP, Keohan ML Combined KIT and CTLA- 4 blockade in patients with refractory GIST and other advanced sarcomas. J. Clin. Oncol. 32(Suppl. 5), Abstract 10521 (2014).

13 von Mehren M, Tetzlaff ED, Macaraeg M. Dose escalating study of crenolanib besylate in advanced GIST patients with PDGFRA D842V activating mutations. J. Clin. Oncol. 34(Suppl.), Abstract 11010 (2016).

14 ClinicalTrials.gov Identifier: NCT02508532. A Phase 1 Study of BLU-285 in Patients With Gastrointestinal Stromal Tumors (GIST) and Other Relapsed and Refractory Solid Tumors. https://clinicaltrials.gov

- Addresses the clinical need of finding $K I T$ D816VIPDGFRA D842V-mutant-specific agents.

15 ClinicalTrials.gov Identifier: NCT01735968. A Dose-Finding Phase Ib Multicenter Study of Imatinib in Combination With the Oral Phosphatidyl-Inositol 3-Kinase (PI3K) Inhibitor BYL719 in Patients With Gastrointestinal Stromal Tumor (GIST) Who Failed Prior Therapy With Imatinib and Sunitinib.

https://clinicaltrials.gov

16 ClinicalTrials.gov Identifier: NCT01468688. A Multi-Arm Dose-Finding Phase Ib Multicenter Study of Imatinib in Combination With the Oral PhosphatidylInositol 3-Kinase (PI3-K) Inhibitor BKM120 in patients with gastrointestinal stromal tumor (GIST) who failed prior therapy with imatinib and sunitinib. https://clinicaltrials.gov

17 Blay J, Labouret NH, Cropet C et al. Biomarker-driven access to vemurafenib in $B R A F$-positive cancers: Second study of the French National AcSé Program. J. Clin. Oncol. 34(Suppl.), Abstract TPS11620 (2016).
18 Chi P. A Phase Ib/II study of MEK162 (binimetinib) in combination with imatinib in patients with advanced gastrointestinal stromal tumor (GIST). J. Clin. Oncol. 33(Suppl.), Abstract 10507 (2015).

19 ClinicalTrials.gov Identifier: NCT02342600 SARC029. Phase II Pilot study of trametinib in combination with pazopanib in patients with metastatic or local-regionally recurrent GIST (gastrointestinal stromal tumors) refractory or intolerant to at least imatinib and sunitinib. https://clinicaltrials.gov

20 Nokhara H, Yamamoto N, Nakamichi S et al. A Phase 1 study of cabozantinib in japanese patients with advanced solid tumors: anti-tumor activity in NSCLC and GIST. Ann. Oncol. 24(Suppl. 9), ix48 (2013).

21 ClinicalTrials.gov Identifier: NCT02216578. Phase II study of cabozantinib in patients with gastrointestinal stromal tumors (GIST) who progressed during neoadjuvant, adjuvant or palliative therapy with imatinib and sunitinib. https://clinicaltrials.gov

22 ClinicalTrials.gov Identifier: NCT02257541. A Phase Ib/II study of BGJ398 in combination with imatinib mesylate in patients with untreated advanced gastrointestinal stromal tumors (GIST). https://clinicaltrials.gov

23 Mehren M, George S, Heinrich MC et al. Results of SARC 022, a Phase II multicenter study of linsitinib in pediatric and adult wild-type (WT) gastrointestinal stromal tumors (GIST). J. Clin. Oncol. 32(Suppl.), Abstract 10507 (2014).

24 Shapiro GI, Kwak E, Dezube BJ et al. First-in-human Phase I dose escalation study of a second generation non-ansamycin HSP90 inhibitor, AT13387, in patients with advanced solid tumors. Clin. Cancer Res. 21(1), 87-97 (2015).

25 ClinicalTrials.gov Identifier: NCT01294202. An open-label, randomised, multi-centre, Phase II study to investigate the safety and efficacy of AT13387, either as monotherapy or in combination with imatinib, in patients with unresectable and/or metastatic malignant GIST whose tumour has progressed following treatment with a maximum of three tyrosine kinase inhibitors. https://clinicaltrials.gov

26 Reilley M, Bailey AM, Subbiah V et al. Phase I clinical trial of combination imatinib and ipilimumab in patients with advanced malignancies. J. Clin. Oncol. 34(Suppl.), Abstract 3054 (2016). 
27 ClinicalTrials.gov Identifier: NCT01907607. Efficacy and safety of PD-0332991 in patients with advanced gastrointestinal stromal tumors refractory to imatinib and sunitinib: a Phase 2 study.

https://clinicaltrials.gov

28 Bauer S, Joensuu H. Emerging agents for the treatment of advanced, imatinib-resistant gastrointestinal stromal tumors: current status and future directions. Drugs 75(12), 1323-1334 (2015).

29 Garner AP, Gozgit JM, Anjum R et al. Ponatinib inhibits polyclonal drug-resistant KIT oncoproteins and shows therapeutic potential in heavily pretreated gastrointestinal stromal tumor (GIST) patients. Clin. Cancer Res. 20 (22), 5745-5755 (2014).

30 Heinrich MC, Mehren M, Demetri GD et al. Ponatinib efficacy and safety in patients (pts) with advanced gastrointestinal stromal tumors (GIST) after tyrosine kinase inhibitor (TKI) failure: results from a Phase 2 study. J. Clin. Oncol. 33(Suppl.), Abstract 10535 (2015).

- Shows promising results with ponatinib.

31 Heinrich MC, Wise S, Hood M et al. In vitro activity of novel KIT/PDGFRA switch pocket kinase inhibitors against mutations associated with drug-resistant GI stromal tumors.

J. Clin. Oncol. 28(Suppl. 15), Abstract 10007 (2010).

32 ClinicalTrials.gov Identifier: NCT02164240 Phase Ib Study of SUnitinib Alternating With REgorafenib in Patients With Metastatic and/or Unresectable GIST (SURE). https://clinicaltrials.gov

33 ClinicalTrials.gov Identifier: NCT02365441. A Randomised Phase II Trial of Imatinib Alternating With Regorafenib Compared to Imatinib Alone for the First Line Treatment of Advanced Gastrointestinal Stromal Tumour (GIST). https://clinicaltrials.gov

34 ClinicalTrials.gov Identifier: NCT01316263. A Phase 2 Study of a Human Anti-PDGFR $\alpha$ Monoclonal Antibody (IMC-3G3) in Previously Treated Patients With Unresectable and/or Metastatic Gastrointestinal Stromal Tumors (GIST). https://clinicaltrials.gov

35 Dewaele B, Wasag B, Cools J et al. Activity of dasatinib, a dual SRC/ABL kinase inhibitor, and IPI-504, a heat shock protein 90 inhibitor, against gastrointestinal stromal tumor-associated PDGFRAD842V mutation. Clin. Cancer Res. 14(18), 5749-5758 (2008).

36 Heinrich MC, Griffith D, McKinley A et al. Crenolanib inhibits the drug-resistant
PDGFRA D842V mutation associated with imatinib-resistant gastrointestinal stromal tumors. Clin. Cancer Res. 18(16), 4375-4384 (2012).

37 Evans Erica K., Hodous Brian L et al. BLU-285, the first selective inhibitor of PDGFR $\alpha$ D842V and KIT exon 17 mutants. Cancer Res. 75 (15 Suppl.), Abstract 791 (2015).

38 Gebreyohannes YK, Zhai ME, Wozniak A et al. Efficacy of BLU-285, a novel, potent inhibitor of exon 17 mutant KIT and PDGFRA D842V, in patient-derived xenograft model of gastrointestinal stromal tumor (GIST). J. Clin. Oncol. 34(Suppl.), Abstract 11030 (2016).

39 Li B, Garcia CS, Marino-Enriquez A et al. Conjoined hyperactivation of the RAS and PI3K pathways in advanced GIST. J. Clin. Oncol. 34(Suppl.), Abstract e22520 (2016).

40 Van Looy T, Wozniak A, Floris G, Sciot R et al. Phosphoinositide 3-kinase inhibitors combined with imatinib in patient-derived xenograft models of gastrointestinal stromal tumors: rationale and efficacy. Clin. Cancer Res. 20(23) 6071-6082 (2014).

41 Agaram NP, Wong GC, Guo T et al. Novel V600E $B R A F$ mutations in imatinib-naive and imatinib-resistant gastrointestinal stromal tumors. Genes Chromosomes Cancer 47(10), 853-859 (2008).

42 Falchook G, Trent J, Heinrich M et al. BRAF mutant gastrointestinal stromal tumor: first report of regression with BRAF inhibitor dabrafenib (GSK2118436) and whole exomic sequencing for analysis of acquired resistance. Oncotarget 4, 310-315 (2013).

43 Chi P, Chen Y, Zhang L et al. ETV1 is a lineage survival factor that cooperates with KIT in gastrointestinal stromal tumours. Nature 467, 849-853 (2010).

44 Ran L, Sirota I, Cao Z et al. Combined inhibition of map kinase and KIT signaling synergistically destabilizes ETV1 and suppresses GIST tumor growth. Cancer Discov. 5, 304-315 (2015).

45 Hayashi Y, Bardsley MR, Toyomasu Y et al. Platelet-derived growth factor receptor-a regulates proliferation of gastrointestinal stromal tumor cells with mutations in KIT by stabilizing ETV1 gastroenterology 149, 420-432 (2015).

46 Cohen NA, Zeng S, Seifert AM et al. Pharmacological inhibition of KIT activates MET signaling in gastrointestinal stromal tumors. Cancer Res. 75 (10), 2061-2070 (2015).

47 Li F, Huynh H, Li X et al. FGFR-mediated reactivation of MAPK signaling attenuates antitumor effects of imatinib in gastrointestinal stromal tumors. Cancer Discov. 5(4) 438-451 (2015).

48 Lasota J, Wang Z, Kim SY et al. Expression of the receptor for type I insulin-like growth factor (IGF1R) in gastrointestinal stromal tumors: an immunohistochemical study of 1078 cases with diagnostic and therapeutic implications. Am. J. Surg. Pathol. 37(1), 114-119 (2013).

49 Gill AJ. Succinate dehydrogenase (SDH) and mitochondrial driven neoplasia. Pathology 44, 285-292 (2012).

50 Marino-Enriquez A, Ou WB, Cowley G et al. Genome-wide functional screening identifies CDC37 as a crucial HSP90-cofactor for KIT oncogenic expression in gastrointestinal stromal tumors. Oncogene 33(14), 1872-1876 (2014).

51 Borghaei H, Paz-Ares L, Horn L et al. Nivolumab versus docetaxel in advanced nonsquamous non-small-cell lung cancer. N. Engl. J. Med. 373(17), 1627-3169 (2015).

52 Robert C, Schachter J, Long GV et al. KEYNOTE-006 investigators.

Pembrolizumab versus ipilimumab in advanced melanoma. N. Engl. J. Med. 372(26), 2521-2532 (2015).

53 Bertucci F, Finetti P, Mamessier E et al. PDL1 expression is an independent prognostic factor in localized GIST. Oncoimmunology 4(5), e1002729 (2015).

54 ClinicalTrials.gov Identifier: NCT02301039. SARC028: a Phase II study of the anti-PD1 antibody pembrolizumab (MK-3475) in patients with advanced sarcomas. https://clinicaltrials.gov

55 Balachandran VP, Cavnar MJ, Zeng S et al. Imatinib potentiates antitumor $\mathrm{T}$ cell responses in gastrointestinal stromal tumor through the inhibition of IDO. Nat. Med. 17(9), 1094-1100 (2011).

56 Schneider-Stock R, Boltze C, Lasota J et al. High prognostic value of p16INK4 alterations in gastrointestinal stromal tumors. J. Clin. Oncol. 21(9), 1688-1697 (2003).

57 Lagarde P, Pérot G, Kauffmann A et al. Mitotic checkpoints and chromosome instability are strong predictors of clinical outcome in gastrointestinal stromal tumors. Clin. Cancer Res. 18(3), 826-838 (2012).

58 Konecny GE, Winterhoff B, Kolarova T et al. Expression of $\mathrm{p} 16$ and retinoblastoma determines response to CDK4/6 inhibition in ovarian cancer. Clin. Cancer Res. 17(6), 1591-1602 (2011). 


\section{REVIEW Szucs, Thway, Fisher et al.}

59 Kang G, Bae BN, Sohn BS et al. Detection of KIT and PDGFRA mutations in the plasma of patients with gastrointestinal stromal tumor. Target Oncol. 10(4), 597-601 (2015).
60 Demetri GD, Jeffers M, Reichardt P et al. Mutational analysis of plasma DNA from patients (pts) in the Phase III GRID study of regorafenib (REG) versus placebo (PL) in tyrosine kinase inhibitor (TKI)-refractory GIST: correlating genotype with clinical outcomes. J. Clin. Oncol. 31, 10503 (2013). 